\title{
Anatomy of quantum critical wave functions in dissipative impurity problems
}

DOI:

10.1103/PhysRevB.95.085104

\section{Document Version}

Final published version

Link to publication record in Manchester Research Explorer

\section{Citation for published version (APA):}

Blunden-Codd, Z., Bera, S., Bruognolo, B., Linden, N-O., Chin, A. W., von Delft, J., Nazir, A., \& Florens, S. (2017). Anatomy of quantum critical wave functions in dissipative impurity problems. Physical Review B: covering condensed matter and materials physics. https://doi.org/10.1103/PhysRevB.95.085104

\section{Published in:}

Physical Review B: covering condensed matter and materials physics

\section{Citing this paper}

Please note that where the full-text provided on Manchester Research Explorer is the Author Accepted Manuscript or Proof version this may differ from the final Published version. If citing, it is advised that you check and use the publisher's definitive version.

\section{General rights}

Copyright and moral rights for the publications made accessible in the Research Explorer are retained by the authors and/or other copyright owners and it is a condition of accessing publications that users recognise and abide by the legal requirements associated with these rights.

\section{Takedown policy}

If you believe that this document breaches copyright please refer to the University of Manchester's Takedown Procedures [http://man.ac.uk/04Y6Bo] or contact uml.scholarlycommunications@manchester.ac.uk providing relevant details, so we can investigate your claim.

\section{OPEN ACCESS}




\title{
Anatomy of quantum critical wave functions in dissipative impurity problems
}

\author{
Zach Blunden-Codd, ${ }^{1,2}$ Soumya Bera, ${ }^{3}$ Benedikt Bruognolo, ${ }^{4,5}$ Nils-Oliver Linden, ${ }^{4}$ Alex W. Chin, ${ }^{6}$ \\ Jan von Delft, ${ }^{4}$ Ahsan Nazir, ${ }^{1}$ and Serge Florens ${ }^{7}$ \\ ${ }^{1}$ Photon Science Institute \& School of Physics and Astronomy, University of Manchester, Oxford Road, Manchester M13 9PL, United Kingdom \\ ${ }^{2}$ Department of Physics, Imperial College London, London SW7 2AZ, United Kingdom \\ ${ }^{3}$ Max-Planck-Institut fuer Physik Komplexer Systeme, 01187 Dresden, Germany \\ ${ }^{4}$ Physics Department, Arnold Sommerfeld Center for Theoretical Physics, and Center for NanoScience, Ludwig-Maximilians-Universiät, \\ Theresienstrasse 37, 80333 München, Germany \\ ${ }^{5}$ Max-Planck-Institut ur Quantenoptik, Hans-Kopfermann-Strasse 1, 85748 Garching, Germany \\ ${ }^{6}$ Theory of Condensed Matter Group, University of Cambridge, J. J. Thomson Avenue, Cambridge, CB3 OHE, United Kingdom \\ ${ }^{7}$ Institut Néel, CNRS and Université Grenoble Alpes, F-38042 Grenoble, France
}

(Received 7 August 2016; published 2 February 2017)

\begin{abstract}
Quantum phase transitions reflect singular changes taking place in a many-body ground state; however, computing and analyzing large-scale critical wave functions constitutes a formidable challenge. Physical insights into the sub-Ohmic spin-boson model are provided by the coherent-state expansion (CSE), which represents the wave function by a linear combination of classically displaced configurations. We find that the distribution of low-energy displacements displays an emergent symmetry in the absence of spontaneous symmetry breaking while experiencing strong fluctuations of the order parameter near the quantum critical point. Quantum criticality provides two strong fingerprints in critical low-energy modes: an algebraic decay of the average displacement and a constant universal average squeezing amplitude. These observations, confirmed by extensive variational matrix-product-state (VMPS) simulations and field theory arguments, offer precious clues into the microscopics of critical many-body states in quantum impurity models.
\end{abstract}

DOI: 10.1103/PhysRevB.95.085104

\section{INTRODUCTION}

The understanding of critical phenomena in classical mechanics owes a great deal to the spatial representation of critical states, whereby the order parameter experiences statistical fluctuations on all length scales due to a diverging correlation length $[1,2]$ at the critical temperature. This scale invariance property was the starting point for one of the most powerful tools in theoretical physics, the renormalization group, which allowed rationalization of classical criticality in terms of trajectories in the space of coupling constants [3]. Today, one frontier of research in critical phenomena lies in the quantum realm, where criticality may govern some of the most fascinating and complex properties found in strongly correlated materials or cold atoms [4,5]. One very fruitful approach is to consider quantum criticality in light of an effective classical theory in higher dimensions [5], combining spatial and temporal fluctuations within the path integral formalism. Quantum phase transitions are then probed through physical response functions that display a diverging correlation length in space-time. However, this point of view does not provide a full picture of the physics at play, especially since quantum criticality pertains to a singular change in a many-body ground state. Developing wave-function-based approaches to strong correlations is indeed a blossoming field, ranging from quantum chemistry [6] to quantum information $[7,8]$, so that hopes are high that quantum critical states may be rationalized in a simpler way.

Our aim in this article is to directly study the quantum critical wave function of a simple toy model, the sub-Ohmic spin-boson Hamiltonian, and to unveil some salient fingerprints of criticality in its ground state. In this standard model, to be described in further detail below, a single quantized spin interacts with a continuum of bosonic modes, with a spectrum of coupling constants that vanishes with a power law $s<1$ at low energy. For this purpose, we shall use a combination of two numerically exact wave-function-based methods for quantum impurity models: a variational matrix-product-state approach (VMPS) [8-11] and the coherent-state expansion (CSE) [12-15]. VMPS is an abbreviation for the variational matrix-product-state (MPS) formulation of the density matrix renormalization group (DMRG), which has been established as a very powerful and flexible technique, also in the context of bosonic impurity models [11,16,17], and will be used as a reference. Its all-purpose character makes it hard, however, to rationalize the precise content of the wave function in simple physical terms. For this reason, we implement the CSE variationally, which amounts to expanding environmental states of the bath onto a discrete set of classical-like configurations, namely, coherent states of the bosonic states in the bath. (Note that an infinite discrete set is enough to ensure completeness of the coherent-state basis [18].) Thus, crucial aspects of quantum criticality can be directly inferred by reading-off the various superpositions of oscillator displacements that parametrize the set of coherent states.

For a given spin orientation of the impurity, we find that the distribution of displacements within the CSE wave function displays an emergent symmetry (between positive and negative values) in the critical domain. This implies that the average displacement decays to zero for low-energy critical modes, with a universal exponent controlled by the dynamical susceptibility. This behavior reflects the absence of spontaneous symmetry breaking and the fact that the magnetization order parameter directly couples to the bosonic displacement field. Hence the displacements of the oscillators in the critical many-body wave function vanish in average 
at low energy. In addition, the CSE wave function indicates that the distribution of displacements admits a finite width at the quantum critical point (although its mean value vanishes algebraically for critical modes, as mentioned above). This observation translates physically the wide fluctuations of the order parameter that take place in the quantum critical regime in absence of ordering. At the level of the critical wave function, these effects amount to a finite average squeezing amplitude of the quantum critical modes (averaged over a logarithmic energy interval), which we show from field theory arguments to take a constant universal value.

For the spin-boson model, we demonstrate that both the MPS and CSE methodologies converge to the same results, both away from and at the critical point. We find that the number of coherent states required to capture quantum critical behavior on a reasonable energy range (at least three decades) is relatively large, of the order of a hundred. For this reason, recent investigations of the sub-Ohmic model with variational CSEs using fewer states [19,20] failed to grasp the critical exponents found in large-scale VMPS calculations [16,17]. In contrast to the usual Kondo problem associated with the Ohmic spin-boson model, the sub-Ohmic case is indeed governed by two energy scales in its delocalized phase, namely, the renormalized tunneling amplitude and the mass of a soft bosonic collective mode which drives the transition. Capturing the critical softening requires careful and extensive numerical simulations, as we shall show by benchmarking the VMPS and CSE against each other.

The paper is organized as follows. In Sec. II, we present the spin-boson model, its discretization on a Wilson energy mesh, and the variational solution of its many-body wave function using both MPS and CSE representations. The wave function obtained by CSE is displayed to guide physical intuition in the rest of the paper. Section III develops the necessary analytical work that relates the dynamical critical exponent of the spin susceptibility to two important features of the wave function: the average displacement of the environmental state and the average width (or squeezing amplitude). This allows us to elucidate the different behaviors of the wave function in both the noncritical delocalized phase and at the quantum critical point. Finally, Sec. IV shows numerical results from the VMPS and CSE approaches, finding excellent agreement between each other, as well as with analytical predictions. Appendix A provides details on our hierarchical algorithm devised to solve the CSE in a fast and reliable way.

\section{GROUND-STATE WAVE FUNCTION OF THE SUB-OHMIC SPIN-BOSON MODEL}

\section{A. Model}

Our study will be based on the spin-boson Hamiltonian [4,21-24] with $\Delta$ the quantum Larmor frequency of a two-level system described by Pauli matrices $\vec{\sigma}$ :

$$
H=\frac{\Delta}{2} \sigma_{x}-\frac{\sigma_{z}}{2} \sum_{k} g_{k}\left(a_{k}^{\dagger}+a_{k}\right)+\sum_{k} \omega_{k} a_{k}^{\dagger} a_{k} .
$$

The bosonic spectrum assumes a pure power law with exponent $0 \leqslant s \leqslant 1$ up to a sharp high-energy cutoff $\omega_{c}\left(\omega_{c}=1\right.$ in all our numerical computations):

$$
J(\omega) \equiv \sum_{k} \pi g_{k}^{2} \delta\left(\omega-\omega_{k}\right)=2 \pi \alpha \omega_{c}^{1-s} \omega^{s} \theta(\omega) \theta\left(\omega_{c}-\omega\right) .
$$

The Ohmic case $(s=1)$ can be realized in the context of waveguide QED [25-28] by coupling a superconducting qubit to a high-impedance transmission line consisting of a uniform Josephson junction array. In principle, a precise tailoring of the capacitance network could allow the sub-Ohmic regime to be realized as well. In terms of quantum critical phenomena [29-33], the sub-Ohmic model with $0 \leqslant s<1$ presents a continuous quantum phase transition at a critical coupling $\alpha_{c}$ between a localized phase (with $\left\langle\sigma_{z}\right\rangle \neq 0$ for $\alpha>\alpha_{c}$ ) and a symmetric phase (with $\left\langle\sigma_{z}\right\rangle=0$ for $\alpha \leqslant \alpha_{c}$ ), which will be our focus.

\section{B. Wilson discretization}

The bosonic bath $J(\omega)$ is discretized in a logarithmic fashion using a Wilson parameter $\Lambda>1$, first on the highest energy window close to the cutoff $\left[\Lambda^{-1} \omega_{c}, \omega_{c}\right]$, and then iteratively on successive decreasing energy intervals $\left[\omega_{n+1}, \omega_{n}\right]$ with $\omega_{n}=\Lambda^{-n} \omega_{c}[16,29,30]$. This leads to the so-called star Hamiltonian, which involves the direct coupling of the spin to all bosonic modes (and not to a single site within an extended bosonic chain):

$$
H_{\text {star }}=\frac{\Delta}{2} \sigma_{x}-\frac{1}{2} \sigma_{z} \sum_{n=0}^{+\infty} \frac{\gamma_{n}}{\sqrt{\pi}}\left[a_{n}^{\dagger}+a_{n}\right]+\sum_{n=0}^{+\infty} \xi_{n} a_{n}^{\dagger} a_{n} .
$$

The impurity coupling strength reads

$$
\gamma_{n}^{2}=\int_{\omega_{n+1}}^{\omega_{n}} d \omega J(\omega)=2 \pi \alpha \frac{1-\Lambda^{-(s+1)}}{s+1} \omega_{c}^{2} \Lambda^{-n(s+1)},
$$

and the typical energy $\xi_{n}$ in each Wilson shell is

$$
\xi_{n}=\frac{1}{\gamma_{n}^{2}} \int_{\omega_{n+1}}^{\omega_{n}} d \omega \omega J(\omega)=\frac{s+1}{s+2} \frac{1-\Lambda^{-(s+2)}}{1-\Lambda^{-(s+1)}} \omega_{c} \Lambda^{-n} .
$$

Note that the continuum limit is only recovered for $\Lambda \rightarrow 1$ and an infinite number of Wilson shells. However, in practice $\Lambda=$ 2 will be used in the following, and 50 sites will be used for both the MPS and the CSE variational calculations. This standard choice of parameters offers a good compromise between energy resolution and numerical costs, but our techniques can be pushed in principle to smaller $\Lambda$ values.

\section{Variational matrix-product-states approach}

One very successful approach that enables direct access to the ground-state wave function of a low-dimensional quantum system is the density matrix renormalization group (DMRG) $[9,10]$. Though originally developed in the context of one-dimensional real-space systems, the matrix-product-state formulation of this variational method (VMPS) has been established as indispensable tool also in the context of quantum impurity models $[8,11,16,17]$.

Its application to the spin-boson model works as follows. First, the star Hamiltonian $H_{\text {star }}$ is mapped on a truncated 
Wilson chain, where the spin- $\frac{1}{2}$ impurity is coupled to a length$N$ tight-binding chain model whose hopping matrix elements decrease exponentially with site number $k$. Next, one initializes a random MPS for the Wilson chain Hamiltonian,

$$
|\psi\rangle=\sum_{\sigma, \mathbf{m}} A^{[\sigma]} A^{\left[m_{0}\right]} A^{\left[m_{1}\right]} \ldots A^{\left[m_{N}\right]}|\sigma\rangle|\mathbf{m}\rangle,
$$

where $|\uparrow\rangle,|\downarrow\rangle$ represents the $\sigma_{z}$ eigenstates of the impurity and $|\mathbf{m}\rangle=\left|m_{0}\right\rangle \ldots\left|m_{N}\right\rangle$ describes boson number eigenstates in a truncated Fock basis, i.e., $\hat{m}_{k}|\mathbf{m}\rangle=m_{k}|\mathbf{m}\rangle$, with $m_{k}=$ $0,1, \ldots, d_{k}-1$. The wave-function coefficient is split into a product of tensors $A^{[\cdots]}$, which are iteratively varied with respect to the energy for finding the best approximation for the ground-state wave function. If the parameters such as the bond dimension $D$ and the Fock-space dimension $d_{k}$ are chosen appropriately large, the algorithm converges the MPS to a numerically quasiexact representation of the ground-state wave function. In practice, we use an optimal boson basis $[16,17]$ mapping the local Fock basis $\left|m_{k}\right\rangle$ to a smaller, effective bosonic basis $\left|\tilde{m}_{k}\right\rangle$ for efficiency reasons. Good convergence is ensured for the delocalized phase and at the quantum critical point for $D=60, d_{k}=100$, and $\tilde{d}_{k}=16$.

\section{Coherent-state expansion}

\section{General methodology}

More recently, an alternative representation of bosonic environmental wave functions was proposed [12,13] based on a simple physical picture of the energy landscape in terms of classical-like configurations. These are parametrized by multimode coherent states, $\left| \pm f^{(m)}\right\rangle=e^{ \pm \sum_{k} f_{k}^{(m)}\left(a_{k}^{\dagger}-a_{k}\right)}|0\rangle$, with $f_{k}^{(m)}$ the displacement of mode $k$ for the $m$ th variational coherent state. Note that the index $k$ labels momentum, while the index $m=1 \ldots M_{\mathrm{cs}}$ represents an optimal choice of a set of discrete coherent states which embodies a complete basis for an infinite number of coherent states, $M_{\mathrm{cs}} \rightarrow \infty$ [18]. The expansion for the many-body ground-state wave function $|\mathrm{GS}\rangle$ reads

$$
|\mathrm{GS}\rangle=\sum_{m=1}^{M_{\mathrm{cs}}}\left[p_{m}\left|f^{(m)}\right\rangle|\uparrow\rangle+q_{m}\left|h^{(m)}\right\rangle|\downarrow\rangle\right],
$$

with the normalization $\langle\mathrm{GS} \mid \mathrm{GS}\rangle=1$. Here, $p_{m}$ and $q_{m}$ characterize the weight of the different coherent-state components within the ground-state wave function for each spin orientation. The discrete sum over the index $m$ can thus be interpreted as an optimal discretization of the multidimensional integral involved in the standard overcomplete Glauber-Sudarshan representation [18] in terms of continuously varying displacement functions. We find in practice that the coherent-state representation does not show signs of this overcompleteness once the wave function is developed on a discrete sum of coherent states, as in Eq. (7), and if the number of coherent states $M_{\mathrm{cs}}$ is typically much less than the number of states in the Hilbert space required to capture the ground state (which corresponds to the usual application of the method). There is, of course, a trivial redundancy when reshuffling the indices $m$ of the set of coherent states for a given solution, but apart from this, we usually find a single global minimum in the variational procedure (although the local minima tend to cluster in energy when more and more states are added). Thus, the full many-body ground state of the spin-boson model can be interpreted physically based on the optimal variational state, a path that we will follow here. We have also developed a hierarchical algorithm for the optimization of the systematic variational state (7), see Appendix A.

For the spin-boson model without any magnetic field along $\sigma_{z}$ and in absence of spontaneous symmetry breaking (which occurs for $\alpha>\alpha_{c}$ ), the system obeys a $\mathbb{Z}_{2}$ symmetry, so that the parameters for the ground state satisfy exactly $p_{m}=-q_{m}$ and $f_{k}^{(m)}=-h_{k}^{(m)}$ for all $k$ and $m$. This method was thoroughly tested for the Ohmic spin-boson model $(s=1)$ [12,13], where extremely rapid convergence was established for a moderate number of coherent states $M_{\mathrm{cs}} \lesssim 10$, unless one considers the deep Kondo regime where $\alpha \rightarrow 1$.

\section{Full many-body wave function}

We show in Fig. 1 typical wave functions obtained with the CSE near the quantum critical point (for two bath exponents $s=0.3$ and $s=0.8$ ). Here the set of displacements $f_{n}^{(m)}$ for each oscillator mode $a_{n}^{\dagger}$ is plotted versus the frequency $\omega_{n}$ of the mode, with $m=1 \ldots M_{\mathrm{cs}}$ the index in the expansion (the corresponding weights $p_{m}$ are shown in the Appendix). In both plots, the critical domain lies roughly for frequencies in the range $10^{-4}<\omega_{n}<10^{-2}$, which shows two striking observations. First, the distribution of displacements looks very symmetric between positive and negative values of the set of $f_{n}^{(m)}$, both in the critical regime, and in the region of runaway
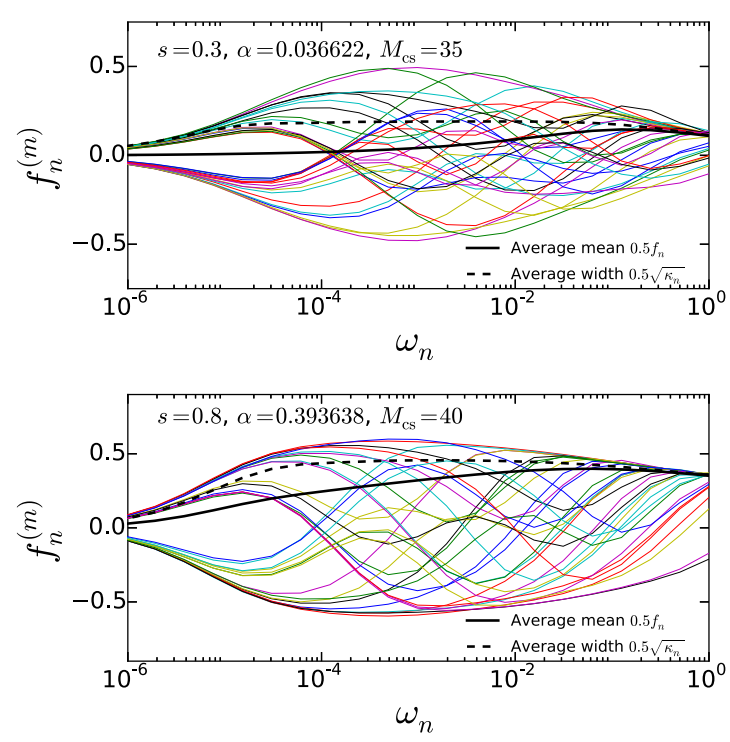

FIG. 1. Nearly critical wave functions from the CSE for the case $s=0.3$ (upper panel) and $s=0.8$ (lower panel), represented by the set of displacements $f_{n}^{(m)}$ with $m=1 \ldots M_{\mathrm{cs}}$ given by the thin full lines. Thick full lines show the average mean displacement $0.5 f_{n}$, and thick dashed lines the average width $0.5 \sqrt{\kappa_{n}}$. The critical regime is identified in the range $10^{-4}<\omega_{n}<10^{-2}$ by a constant plateau in $\kappa_{n}$, which reflects the clearly wide distribution of the displacements associated to the classical-like configurations of the CSE. For frequencies $\omega \ll 10^{-4}$, the wave function is no more critical and the displacements collapse onto a single curve, so that the distribution narrows, and $\kappa_{n}$ goes to zero. 
flow $\omega_{n}<10^{-4}$ at lower energy. This symmetry is clearly not obeyed for the high-energy modes near the cutoff. Because the displacement operator directly couples to the order parameter $\sigma_{z}$ in the Hamiltonian (1), this symmetry nicely reflects the absence of spontaneous symmetry breaking at the critical point. This observation can be substantiated mathematically by defining, from the star Hamiltonian (3), the average $f_{n}$ of the displacement fields in mode $n$ (see Sec. III for a thorough discussion):

$$
\begin{aligned}
f_{n} & \equiv\left\langle\left(a_{n}^{\dagger}+a_{n}\right) \sigma_{z}\right\rangle \\
& =2 \sum_{m, m^{\prime}}^{M_{C S}} p_{m} p_{m^{\prime}}\left\langle f^{(m)} \mid f^{\left(m^{\prime}\right)}\right\rangle\left(f_{n}^{(m)}+f_{n}^{\left(m^{\prime}\right)}\right) .
\end{aligned}
$$

The absence of spontaneous symmetry breaking, both at the critical point and in the whole delocalized phase, translates in the fact that the average value $f_{n}$ vanishes for $\omega_{n} \rightarrow 0$. However, the set of displacements in the noncritical domain $\left(\omega_{n}<10^{-4}\right)$ obey a trivial symmetry, as all displacements collapse on a single curve. In contrast, the displacements in the critical range $10^{-4}<\omega_{n}<10^{-2}$ keep fluctuating, showing a finite width of the distribution. This width $\kappa_{n}$ can be defined as follows:

$$
\begin{aligned}
\kappa_{n} & \equiv\left\langle\left(a_{n}^{\dagger}+a_{n}\right)^{2}\right\rangle-1 \\
& =2 \sum_{m, m^{\prime}}^{M_{C S}} p_{m} p_{m^{\prime}}\left\langle f^{(m)} \mid f^{\left(m^{\prime}\right)}\right\rangle\left(f_{n}^{(m)}+f_{n}^{\left(m^{\prime}\right)}\right)^{2} .
\end{aligned}
$$

This plateau in $\kappa_{n}$, seen in the critical domain, has for origin the strong quantum fluctuations that take place at criticality due to an order parameter that is nearly but not quite localized. Alternatively, the width $\kappa_{n}$ can be interpreted as a squeezing parameter for the mode $a_{n}^{\dagger}$.

Having clarified the physics at play in the wave function itself, we will study these two coarse-grained quantities $f_{n}$ (average) and $\kappa_{n}$ (width), which capture mathematically the distribution of classical configurations in the wave function. This study will rely not only on the CSE variational state, but also on VMPS calculations for benchmark, and on analytical field theory calculations, which we present now.

\section{ANALYTICAL INSIGHTS INTO VARIOUS WAVE-FUNCTION PROPERTIES}

We establish in this section a set of exact analytical results for various wave-function properties, both in the noncritical and in the critical regimes. The properties that we will consider concern the average displacement of the bath oscillators, as well as their squeezing amplitude, which can be interpreted as the variance of the oscillator displacements. These two quantities thus give interesting information on the structure of the environmental wave function.

\section{A. Average displacement}

\section{General formula}

Owing to the linear coupling between $\sigma_{z}$ and the oscillator displacement operator $\left(a_{k}^{\dagger}+a_{k}\right)$, correlations are established between the spin degree of freedom and its bosonic environ- ment. Due to the symmetry properties of Hamiltonian (1), the ground-state wave function can be written generically as $|\mathrm{GS}\rangle=|\uparrow\rangle\left|\Psi_{\uparrow}\right\rangle-|\downarrow\rangle\left|\Psi_{\downarrow}\right\rangle$, where $\left|\Psi_{\downarrow}\right\rangle=\hat{P}\left|\Psi_{\uparrow}\right\rangle$, with the parity operator $\hat{P}=\exp \left(i \pi \sum_{k} a_{k}^{\dagger} a_{k}\right)$. Thus, except for the trivial noninteracting case $\alpha=0$ where the environmental wave function is in the bare vacuum, the qubit does not factorize from its environment. The manner in which correlations in $\left|\Psi_{\uparrow}\right\rangle$ penetrate the bath states can be viewed equally as properties of a screening cloud [15,28]. One goal of this paper is to illustrate the behavior of this screening cloud in the sub-Ohmic model, both away from and at the quantum critical point. Since the environmental wave function $\left|\Psi_{\uparrow}\right\rangle$ is a complicated object, the simplest measure of the cloud resides in the average displacement $f_{k}$ that is obeyed by a given but arbitrary mode $a_{k}^{\dagger}$ within this state. This quantity is defined as $f_{k} \equiv<\left(a_{k}^{\dagger}+a_{k}\right) \sigma_{z}>$, where the average is taken with respect to the full many-body ground state $|\mathrm{GS}\rangle$. The average displacement $f_{k}$ thus gives information on how strong the order parameter fluctuates at the energy scale $\omega_{k}$.

Now, we would like to show that this average displacement can be related exactly to the spin-spin equilibrium correlation function, defined in imaginary time as

$$
\chi(\tau)=\left\langle\mathrm{GS}\left|T_{\tau} \frac{\sigma_{z}(\tau)}{2} \frac{\sigma_{z}(0)}{2}\right| \mathrm{GS}\right\rangle,
$$

with $T_{\tau}$ the standard time-ordering operator, so that $T_{\tau} A(\tau) B(0)=\theta(\tau) A(\tau) B(0)+\theta(-\tau) B(0) A(\tau)$. The imaginary-time evolved operators $\operatorname{read} A(\tau)=e^{H \tau} A e^{-H \tau}$. For the purpose of computing $f_{k}$, let us introduce the mixed correlation function between the spin and the displacement operator associated to a given bosonic $k$ mode:

$$
G_{z, k}(\tau) \equiv\left\langle\mathrm{GS}\left|T_{\tau}\left[a_{k}^{\dagger}(\tau)+a_{k}(\tau)\right] \sigma_{z}(0)\right| \mathrm{GS}\right\rangle,
$$

so that $f_{k}=G_{z, k}\left(0^{+}\right)$. Taking the time derivative in Eq. (11), one gets the equations of motion:

$$
\frac{\partial^{2}}{\partial \tau^{2}} G_{z, k}(\tau)=\omega_{k}^{2} G_{z, k}(\tau)-4 g_{k} \omega_{k} \chi(\tau) .
$$

Now, going to zero temperature (but the formula below applies as well to finite temperature using discrete Matsubara frequencies), with $G(i \omega)=\int_{-\infty}^{+\infty} d t G(\tau) e^{i \omega t}$, one obtains the exact relation:

$$
G_{z, k}(i \omega)=\frac{4 g_{k} \omega_{k}}{\omega^{2}+\omega_{k}^{2}} \chi(i \omega)
$$

Going back to the time domain, one finds the connection between the average displacement of the environmental wave function (the screening cloud) and the local spin susceptibility:

$$
f_{k}=\int \frac{d \omega}{2 \pi} G_{z, k}(i \omega)=4 g_{k} \omega_{k} \int \frac{d \omega}{2 \pi} \frac{1}{\omega^{2}+\omega_{k}^{2}} \chi(i \omega) .
$$

From this equation, previous knowledge obtained for spin dynamics of the sub-Ohmic model [29,30,33] will allow us to make exact predictions for the average displacement characterizing the screening cloud. 


\section{Asymptotic behavior of the average displacement}

A change of variable in Eq. (14) gives

$$
f_{k}=4 g_{k} \int \frac{d x}{2 \pi} \frac{1}{x^{2}+1} \chi\left(i \omega_{k} x\right)
$$

so that the small-momentum behavior of $f_{k}$ is determined by the low-energy scaling of the spin-spin correlation function $[29,30,33]$, which reads $\chi(i \omega) \simeq 1 /\left(m_{R}+B_{s}|\omega|^{s}\right)$, with $B_{s}=$ $4 \alpha \omega_{c}^{1-s} \int d x x^{s-1} /\left(1+x^{2}\right)$. Here $m_{R}$ is the renormalized mass, which is finite in the delocalized phase $\left(\alpha<\alpha_{c}\right)$ and vanishes at the quantum critical point. Thus, two scaling laws are established in the limit $k \rightarrow 0$ :

$$
\begin{gathered}
f_{k} \simeq \frac{2 g_{k}}{m_{R}} \text { for } \alpha<\alpha_{c}, \\
f_{k} \simeq \frac{4 A_{s}}{B_{s}} \frac{g_{k}}{\left|\omega_{k}\right|^{s}} \text { for } \alpha=\alpha_{c},
\end{gathered}
$$

where $A_{s}=\int(d x / 2 \pi) x^{-s} /\left(1+x^{2}\right)$. Let us now specialize to the case of the Wilson energy discretization on the grid $\omega_{n}=\omega_{c} \Lambda^{-n}$, in which case $\omega_{k}$ is replaced by $\xi_{n} \propto \Lambda^{-n} \propto \omega_{n}$ and $g_{k}$ by $\gamma_{n} / \sqrt{\pi} \propto \Lambda^{-n(s+1) / 2} \propto \omega_{n}^{(s+1) / 2}$. We thus find the following low-energy scaling laws of the average displacement for the modes obeying the Wilson energy discretization:

$$
\begin{aligned}
& f_{n} \propto \omega_{n}^{(1+s) / 2} \text { for } \alpha<\alpha_{c}, \\
& f_{n} \propto \omega_{n}^{(1-s) / 2} \text { for } \alpha=\alpha_{c} .
\end{aligned}
$$

The noncritical modes thus follow a different and faster power law than the critical ones, a result that we shall confirm from our numerics in Sec. IV. In fact, our low-frequency analysis allows us to extract the exact prefactor of the critical average displacement. At $\alpha=\alpha_{c}$, we find

$$
f_{n}=\frac{\sqrt{2}(s+2)^{s}\left(1-\Lambda^{-(s+1)}\right)^{s+\frac{1}{2}} \tan \frac{\pi s}{2}}{\pi \sqrt{\alpha} \omega_{c}^{\frac{1-s}{2}}(s+1)^{s+\frac{1}{2}}\left(1-\Lambda^{-(s+2)}\right)^{s}} \omega_{n}^{\frac{1-s}{2}} .
$$

The prefactor is clearly nonuniversal, as a dependence in the frequency cutoff $\omega_{c}$ is present.

\section{B. Average width (squeezing amplitude)}

\section{General formula}

Generalizing the previous results, we define the average intramode squeezing amplitude as $\kappa_{k} \equiv\left\langle\left(a_{k}^{\dagger}+a_{k}\right)^{2}\right\rangle-1$, such that it is exactly zero for a vacuum state. Following the previous methodology, we introduce the intermode Green's function of the displacement field of the bosonic modes:

$$
G_{k, q}(\tau) \equiv\left\langle\mathrm{GS}\left|T_{\tau}\left[a_{k}^{\dagger}(\tau)+a_{k}(\tau)\right]\left[a_{q}^{\dagger}(0)+a_{q}(0)\right]\right| \mathrm{GS}\right\rangle .
$$

Applying the time derivative twice provides exact equations of motion, which lead to the following formula in the Matsubara domain:

$$
G_{k, q}(i \omega) \equiv G_{k}^{0}(i \omega) \delta_{k, q}+g_{k} g_{q} G_{k}^{0}(i \omega) G_{q}^{0}(i \omega) \chi(i \omega),
$$

where $G_{k}^{0}(i \omega)=2 \omega_{k} /\left(\omega^{2}+\omega_{k}^{2}\right)$. This gives the exact equation relating the average squeezing parameter to the dynamical spin-spin susceptibility:

$$
\kappa_{k}=\int \frac{d \omega}{2 \pi} G_{k, k}(i \omega)-1=4 g_{k}^{2} \omega_{k}^{2} \int \frac{d \omega}{2 \pi} \frac{1}{\left(\omega^{2}+\omega_{k}^{2}\right)^{2}} \chi(i \omega) .
$$

Again, knowledge of the spin dynamics will give information on the average squeezing parameter for the ground-state wave function.

\section{Asymptotic behavior of the average squeezing}

Similar to our analysis of the average displacement, a change of variable in Eq. (23) gives

$$
\kappa_{k}=\frac{4 g_{k}^{2}}{\omega_{k}} \int \frac{d x}{2 \pi} \frac{1}{\left(x^{2}+1\right)^{2}} \chi\left(i \omega_{k} x\right),
$$

resulting in the following low-energy leading-order behavior of the average squeezing amplitude:

$$
\begin{gathered}
\kappa_{k} \simeq \frac{g_{k}^{2}}{m \omega_{k}} \text { for } \alpha<\alpha_{c}, \\
\kappa_{k} \simeq \frac{4 C_{s}}{B_{s}} \frac{g_{k}^{2}}{\left|\omega_{k}\right|^{1+s}} \text { for } \alpha=\alpha_{c},
\end{gathered}
$$

where $C_{s}=\int(d x / 2 \pi) x^{-s} /\left(1+x^{2}\right)^{2}$. In the case of the Wilson energy discretization on the grid $\omega_{n}=\omega_{c} \Lambda^{-n}$, we get the explicit scaling laws for the average squeezing amplitude:

$$
\begin{aligned}
& \kappa_{n} \propto \omega_{n}^{s} \text { for } \alpha<\alpha_{c}, \\
& \kappa_{n}=\text { const. for } \alpha=\alpha_{c} .
\end{aligned}
$$

We find a constant and universal (cutoff independent) value of $\kappa_{n}$ at the quantum critical point, as a precise computation of the constant value for $\alpha=\alpha_{c}$ reads

$$
\kappa_{n}=\frac{(s+2)^{s+1}\left(1-\Lambda^{-(s+1)}\right)^{s+2} \tan \frac{\pi s}{2}}{\pi(s+1)^{s+1}\left(1-\Lambda^{-(s+2)}\right)^{s+1}} .
$$

Since the average displacement $f_{n}$ vanishes at low energy, this means that the distribution of displacements of the critical wave function is very broad, reflecting the strong fluctuations of the order parameter at the quantum critical point. We stress that $\kappa_{n}$, defined as (10), strictly vanishes in the continuum limit $\Lambda \rightarrow 1$ but that it remains finite when integrated over a logarithmic energy mesh.

\section{NUMERICAL RESULTS}

\section{A. General scaling behavior}

We start by presenting general VMPS calculations, allowing us to outline the scaling behavior and the quantum criticality of the sub-Ohmic spin-boson model. We shall consider two different values of the bath spectral density throughout the paper, $s=0.3$ and $s=0.8$. The former corresponds to the case where the quantum phase transition is of mean-field type, while the latter case is associated to an interacting fixed point [29-32]. We stress beforehand that both the average displacement $f_{n}$ and average squeezing amplitude $\kappa_{n}$ are exactly related to the dynamical susceptibility from Eqs. (14) and (23), so that their scaling behavior as a function 

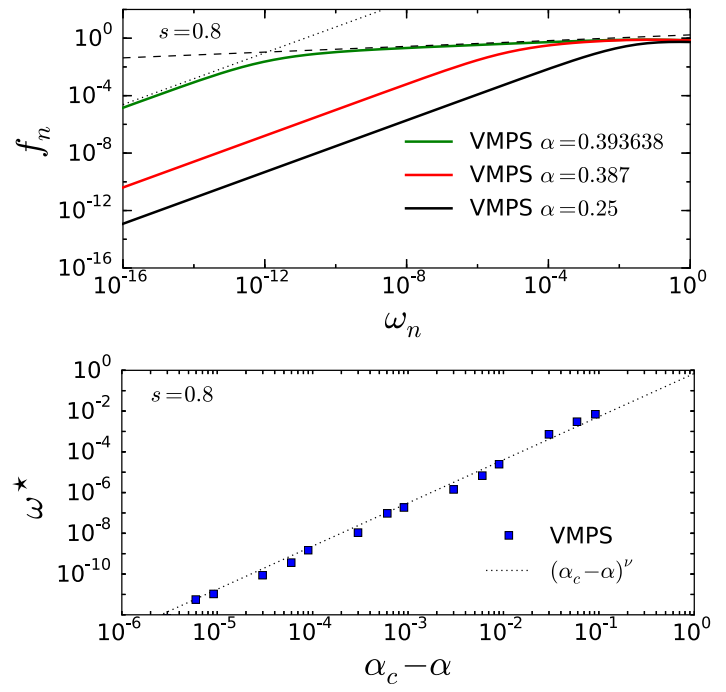

FIG. 2. The upper panel shows the average displacement $f_{n}=$ $\left\langle\left(a_{n}^{\dagger}+a_{n}\right) \sigma_{z}\right\rangle$ from the VMPS calculation at $s=0.8$, for three values of $\alpha=0.25,0.387,0.393638$. (The last value is very close to the quantum critical interaction strength $\alpha_{c}$ ). The dotted line denotes the noncritical scaling $f_{n} \propto \omega_{n}^{(1+s) / 2}$ for $\omega \ll \omega^{\star}$, while the dashed line indicates the expected critical behavior $f_{n} \propto \omega_{n}^{(1-s) / 2}$ for $\omega^{\star} \ll$ $\omega \ll \omega_{c}$. The crossover scale $\omega^{\star}$ between the two scaling behaviors is shown in the lower panel for a large selection of $\alpha$ values, allowing us to extract the correlation length exponent $v \simeq 0.47$ for $s=0.8$. This value is quite different from the mean-field result $v_{\mathrm{MF}}=1 / \mathrm{s}=1.25$, because the system lies below its upper critical dimension $[29,30]$.

of momenta, both in the noncritical and critical regimes, is determined by a trivial $s$-dependent exponent.

However, nontrivial exponents in the interacting case $0.5<$ $s<1$ will show up in the $\alpha$ dependence of the correlation length $\xi$ that is defined by the spatial extent up to which quantum critical fluctuations penetrates within the bath states. More precisely, the correlation length is given by an inverse energy $\xi=1 / \omega^{\star}$, where $\omega^{\star}$ is such that quantum critical behavior is established for $\omega^{\star} \ll \omega_{k} \ll \omega_{c}$. (This regime sets in only if $\alpha$ is quite close to $\alpha_{c}$.) This correlation length behaves as $\xi \propto\left|\alpha_{c}-\alpha\right|^{-v}$, with the exponent $\nu_{\mathrm{MF}}=1 / \mathrm{s}$ in the mean-field regime $0<s<1 / 2$. This can be gathered from the low-energy behavior $\chi(i \omega) \simeq 1 /\left(m_{R}+B_{s}|\omega|^{s}\right)$ and the absence of singular vertex corrections at mean-field level, giving the renormalized mass $m_{R} \propto \alpha_{c}-\alpha$. However, $v$ assumes nontrivial values given by a classical long-range Ising model [29,30] for the interacting regime $1 / 2<s<1$. This behavior is illustrated in the lower panels of Figs. 2 and 3. Thus, both the average displacement and average squeezing amplitude (not shown here) encode nontrivial exponents for $1 / 2<s<1$, but only due to the divergent correlation length $\xi=1 / \omega^{\star}$. These observations can be also summed up by scaling laws:

$$
\begin{gathered}
f_{n}=\omega_{n}^{(1-s) / 2} F\left(\omega_{n} / \omega^{\star}\right), \\
\kappa_{n}=K\left(\omega_{n} / \omega^{\star}\right),
\end{gathered}
$$

with $F(x), K(x) \propto 1$ for $x \gg 1$, and $F(x), K(x) \propto x^{s}$ for $x \ll$ 1. This general scaling behavior of the average displacement
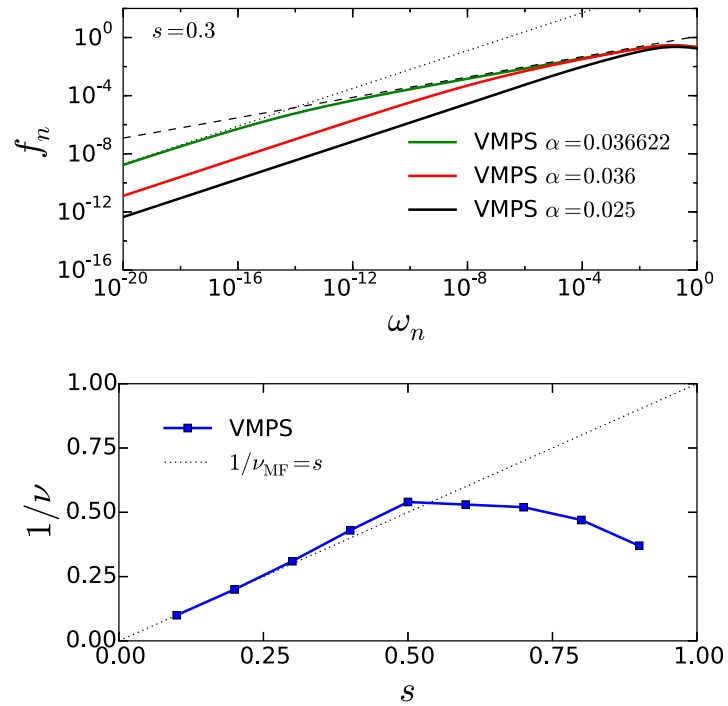

FIG. 3. The upper panel shows, similarly to Fig. 2, the average displacement, but now for $s=0.3$, with $\alpha=0.0326,0.036$, 0.036622 . (The last value is very close to the quantum critical point.) The dotted line shows the noncritical scaling $f_{n} \propto \omega_{n}^{(1+s) / 2}$, while the dashed line indicates the expected critical quantum behavior $f_{n} \propto \omega_{n}^{(1-s) / 2}$. The lower panel shows the extracted correlation length exponent $v$ for various values of $s$, which assumes the mean-field prediction $v_{\mathrm{MF}}=1 / s$ only for $0<s<1 / 2$.

is illustrated in the upper panel of Fig. 2 for $s=0.8$ and in the upper panel of Fig. 3 for $s=0.3$. We find indeed that our VMPS data exhibits the expected noncritical and critical scaling laws, respectively $f_{n} \propto \omega_{n}^{(1+s) / 2}$ for $\alpha \ll \alpha_{c}$ (dotted line) and $f_{n} \propto \omega_{n}^{(1-s) / 2}$ for $\alpha=\alpha_{c}$ (dashed line). We now turn to a more detailed analysis, with a comparison to our analytical predictions, and with the numerics from the coherent-state expansion.

\section{B. Noncritical regime}

Let us now investigate the noncritical regime, which is established either for $\alpha \ll \alpha_{c}$ at all frequencies, or for $\alpha \simeq \alpha_{c}$ but for $\omega \ll \omega^{\star}$. Focusing first on the average displacement, we consider in Fig. 4 the two cases $s=0.3$ and $s=0.8$ for values of $\alpha$ that are sufficiently away from $\alpha_{c}$ so that critical behavior is not triggered. The comparison between the fully converged VMPS data and CSE at increasing number $M_{\mathrm{cs}}$ of coherent states shows that the CSE converges very quickly in this simplest noncritical regime. In addition, the CSE captures the exact leading behavior of the average displacement, $f_{n} \propto \omega_{n}^{(1+s) / 2}$, already for $M_{\mathrm{cs}}=1$ (the so-called Silbey-Harris theory [34-36]), since the variational equation gives $f_{k}=$ $\left(g_{k} / 2\right) /\left(\omega_{k}+\Delta_{R}\right) \propto g_{k} / \Delta_{R}$ for $k \rightarrow 0$, in agreement with the exact result (16). Note that the quantum critical scaling $f_{n} \propto \omega_{n}^{(1-s) / 2}$ is not apparent in this plot because the $\alpha$ value is too far away from $\alpha_{c}$.

Turning to the average squeezing amplitude, we find excellent agreement of our converged CSE results to the expected noncritical scaling behavior $\kappa_{n} \propto \omega_{n}^{s}$ (see Fig. 5). However, we observe a much slower convergence of the CSE for the average squeezing amplitude as compared to 

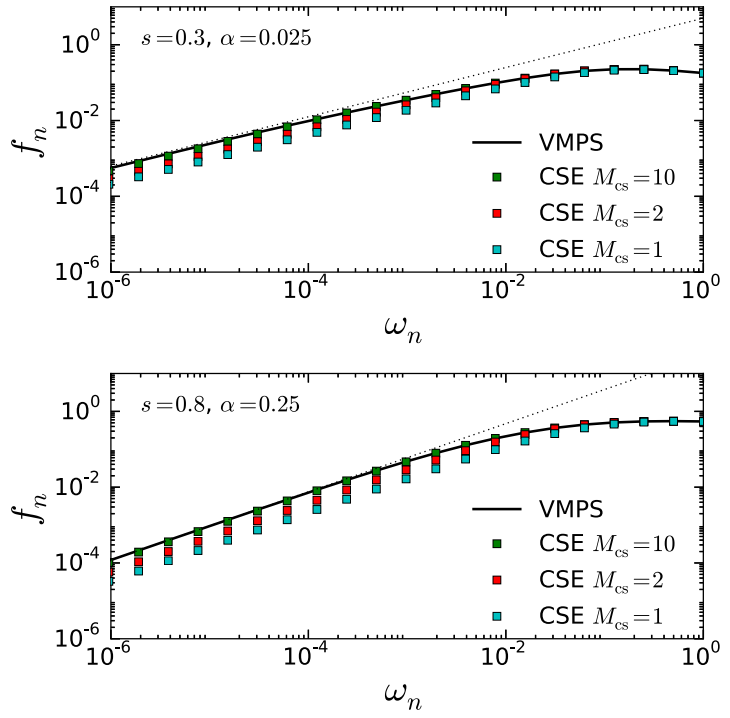

FIG. 4. Average displacement $f_{n}$ of mode $a_{n}^{\dagger}$ in the noncritical regime $\left(\alpha \ll \alpha_{c}\right)$ for two values of the bath spectra, $s=0.3$ (top panel, with $\alpha=0.025$ ) and $s=0.8$ (bottom panel, with $\alpha=0.25$ ). The full black line denotes the fully converged VMPS results, while the colored symbols show the CSE at increasing number of coherent states, $M_{\mathrm{cs}}=1,2,10$ (bottom to top). A dotted line denotes the expected $f_{n} \propto \omega_{n}^{(1+s) / 2}$ behavior in the noncritical regime.

the computation of the average displacements in Fig. 4, especially regarding the low-energy modes. This behavior can be understood from the Silbey-Harris theory at $M_{c s}=1$, which predicts incorrectly $\kappa_{n}=\left(f_{n}\right)^{2} \propto \omega_{n}^{1+s}$ instead of the exact noncritical scaling $\kappa_{n} \propto \omega_{n}^{s}$. This disagreement is not
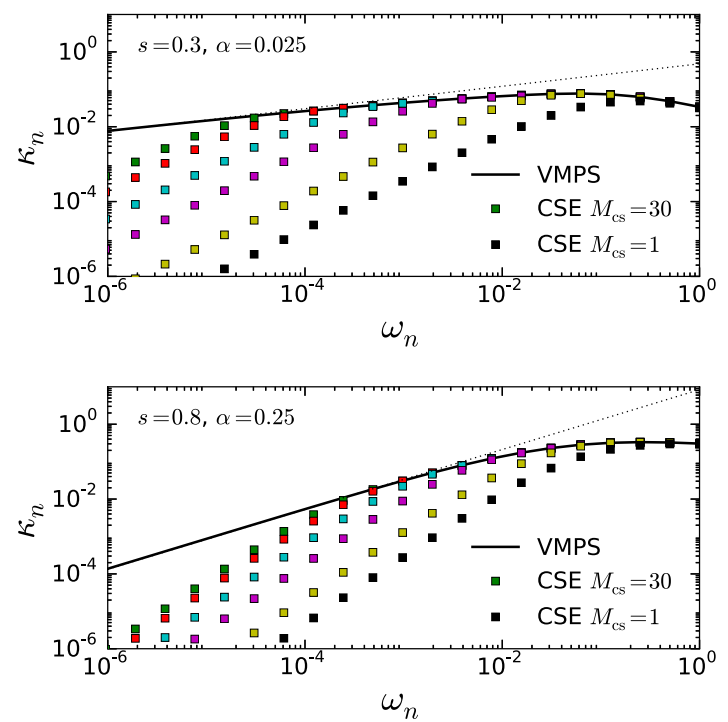

FIG. 5. Average squeezing amplitude $\kappa_{n}$ of mode $a_{n}^{\dagger}$ in the noncritical regime $\left(\alpha \ll \alpha_{c}\right)$ for two values of the bath spectra, $s=0.3$ (top panel, with $\alpha=0.025$ ) and $s=0.8$ (bottom panel, with $\alpha=0.25)$. The full black line denotes the fully converged VMPS results, while the colored symbols show the CSE at increasing number of coherent states, $M_{\mathrm{cs}}=1,2,5,10,20,30$ (bottom to top). A dotted line denotes the expected $\kappa_{n} \propto \omega_{n}^{s}$ behavior in the noncritical regime. fully a surprise, because the Silbey-Harris theory is based on a single coherent state and is tailored to address at best the displacement and not necessarily the squeezing amplitude. As a matter of fact, one can prove from the explicit form of the displacements [13] at arbitrary $M_{\mathrm{cs}}$ values that the incorrect scaling behavior $\kappa_{n} \propto \omega_{n}^{1+s}$ at vanishing $\omega_{n}$ is found for any finite value of $M_{\mathrm{cs}}$, which is also clear from Fig. 5. Only in the strict limit $M_{\mathrm{cs}} \rightarrow \infty$ is the correct noncritical scaling obeyed down to zero energy. Nevertheless, if one focuses on a reasonable energy range (typically a few decades), the correct noncritical scaling behavior is well captured for both the average displacement and the average squeezing amplitude in our CSE computations. This analysis illustrates the general fact that systematic variational calculations may lead to the rapid convergence of some physical observables but not of others. This problem is particularly severe near quantum critical points, because the deviations concern asymptotically low energy modes, which occupy a tiny fraction of the total ground-state energy.

\section{Critical regime}

We now consider the quantum critical point, where the dissipation strength $\alpha=\alpha_{c}$ is such that the correlation length $\xi=1 / \omega^{\star}$ diverges. In practice we fine tune $\alpha_{c}-\alpha$ to more than seven digits so that $\xi$ is larger than $10^{10}$, as can be seen from the VMPS data of Fig. 2. The coherent-state expansion offers, alternatively, a more pictorial view of the quantum critical wave function, which can be fully represented by a set of classical-like displacement configurations, as shown previously in Fig. 1.

While the average critical displacement $f_{n} \propto \omega_{n}^{(1-s) / 2}$ vanishes (with the expected exponent) at low energy, we showed analytically in Eq. (28) that the average squeezing amplitude $\kappa_{n}=\left\langle\left(a_{n}^{\dagger}+a_{n}\right)^{2}\right\rangle-1$ is constant at the quantum critical point. Thus $\kappa_{n}$ can be viewed as the average fluctuation of the displacements within the many-body wave function. Therefore we conclude that $\kappa_{n} \gg\left(f_{n}\right)^{2}$ at the quantum critical point, which reflects the strong fluctuations of the order parameter. This expected physical picture is very clear in Fig. 1: in the intermediate energy range $10^{-4}<\omega<10^{-2}$, the distribution of displacements is nearly symmetric around zero, and thus almost vanishes on average. (This behavior is more pronounced for $s=0.3$ than for $s=0.8$ because the average displacement vanishes as $\omega_{n}^{(1-s) / 2}$.) In contrast, the width of the distribution of displacements has roughly a constant value in the critical domain. Away from the critical domain, namely, for very low frequencies $\omega \ll \omega^{\star}$, the distribution of the classicallike configurations becomes very narrow as all displacements collapse onto the same curve. Thus the average squeezing amplitude should vanish, with the noncritical scaling behavior $\kappa_{n} \propto \omega_{n}^{s}$. However, due to the finite size of the coherent-state basis set used here, we find for this computation the different behavior $\kappa_{n} \propto \omega_{n}^{1+s}$ as discussed previously.

Let us finally check in more detail the precise scaling behavior of the critical average displacement in Fig. 6. Again we find excellent convergence of the CSE to the VMPS curves, and we are able to match quantitatively the expected scaling law Eq. (20), including the analytic prefactor $F_{S}$ in front of 

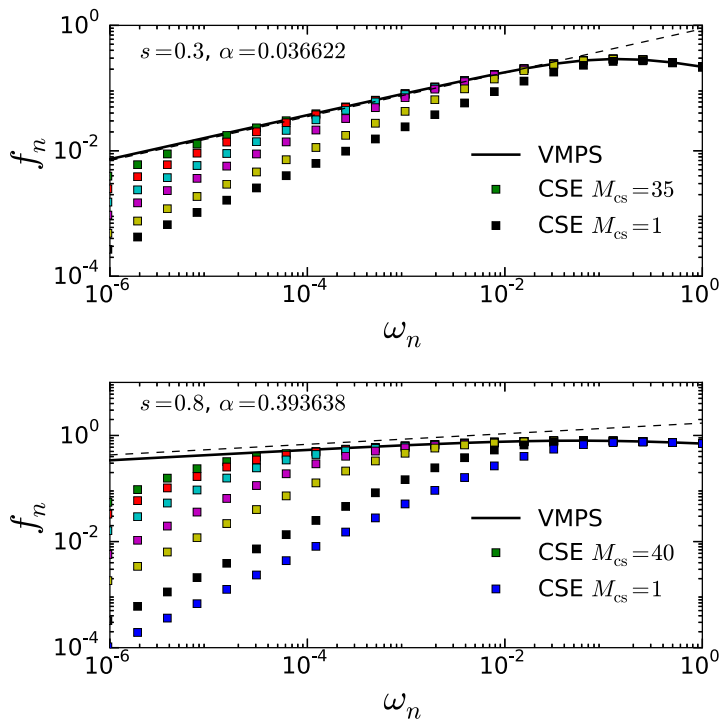

FIG. 6. Average displacement $f_{n}$ of mode $a_{n}^{\dagger}$ at the critical point $\left(\alpha=\alpha_{c}\right.$ ) for two values of the bath spectra, $s=0.3$ (top panel, with $\alpha=0.03662$ ) and $s=0.8$ (bottom panel, with $\alpha=0.393638$ ). The full black line denotes the fully converged VMPS results, while the colored symbols show the CSE at increasing number of coherent states, $M_{\mathrm{cs}}=1,2,5,10,20,35$ (bottom to top) for $s=0.3$ and $M_{\mathrm{cs}}=$ $1,2,5,10,20,30,40$ for $s=0.8$. A dashed line denotes the expected $f_{n} \simeq F_{s} \omega_{n}^{(1-s) / 2}$ scaling behavior in the critical regime, including the analytical prefactor $F_{s}$ given in Eq. (20).

the power law $\omega_{n}^{(1-s) / 2}$. Due to the construction of the CSE based on coherent states, one sees again that any truncation to finite $M_{\mathrm{cs}}$ produces an incorrect scaling $f_{n} \propto \omega_{n}^{(1+s) / 2}$ at
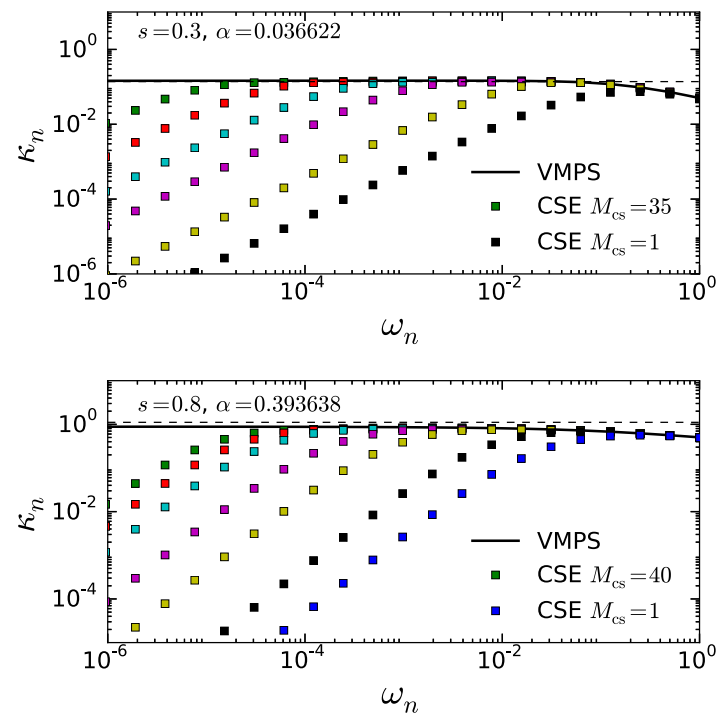

FIG. 7. Average squeezing amplitude $\kappa_{n}$ of mode $a_{n}^{\dagger}$ at the critical point ( $\alpha=\alpha_{c}$ ) for two values of the bath spectra, $s=0.3$ (top panel, with $\alpha=0.03662$ ) and $s=0.8$ (bottom panel, with $\alpha=0.393638$ ). The full black line denotes the fully converged VMPS results, while the colored symbols show the CSE at increasing number of coherent states, $M_{\mathrm{cs}}=1,2,5,10,20,35$ (bottom to top) for $s=0.3$ and $M_{\mathrm{cs}}=$ $1,2,5,10,20,30,40$ for $s=0.8$. A dashed line denotes the expected universal constant value of $\kappa_{n}$ given by Eq. (28) in the critical regime. vanishing energy. But the correct power law is typically obeyed on several decades for a moderate numerical effort. The same type of behavior is also found in the critical average squeezing amplitude $\kappa_{n}$, which shows the expected constant plateau (see Fig. 7) and that matches the analytical prediction of Eq. (28) nicely. We have assessed the general prediction of the power-law dependence of the critical average displacement $f_{n} \propto \omega_{n}^{(1-s) / 2}$ by fitting the low-energy tails of our converged data for a wide selection of the bath exponent $s$ in the range $0<s<1$. We found that the critical exponent $(1-s) / 2$ is very well obeyed, both in the mean field and interacting regimes, with an accuracy of a few percent. This reflects the peculiarity of the spin-boson model, which does not present anomalous exponents in the spin-spin correlation function [29,30,33], even below its upper critical dimension.

\section{CONCLUSION}

We have investigated physical properties of ground-state wave functions in a simple model of quantum criticality, the sub-Ohmic spin-boson Hamiltonian. For this purpose, a combination of variational matrix product states and an extensive coherent-state expansion has been performed and compared very precisely. The coherent-state approach allows a direct representation of many-body wave functions in terms of a collection of classical-like trajectories associated to a set of displacements. Focusing on the quantum critical regime, the wave function displays a nearly symmetric distribution of displacements at low energy. However its width, related to a squeezing amplitude of the low-energy modes defined on a logarithmic energy interval, remains finite with a universal value. This behavior strikingly reflects the wide fluctuations of the order parameter at the quantum critical point in absence of spontaneous symmetry breaking, in analogy with strong statistical fluctuations near classical phase transitions. Detailed analytical predictions have been made using exact field theory results, which match very well all the obtained numerical data, both in the noncritical and critical regimes. Similar analysis should be possible for various extensions of the dissipative impurity model, such as the two-bath case [16,17], which presents new classes of interacting fixed points. It should be applicable also to fermionic models, both with impurities or with bulk interactions, using a similar decomposition of the many-body wave function in terms of a distribution of one- or two-body phase shifts [37].

\section{ACKNOWLEDGMENTS}

We thank H. Baranger, N. Roch, and I. Snyman for useful discussions. S.F., A.N., A.W.C., and Z.B.C. acknowledge funding from the CNRS PICS contract "StrongCircQED" (No. 191148). A.N. and Z.B.C. are also supported by The University of Manchester and the EPSRC. A.W.C. acknowledges support from the Winton Programme for the Physics of Sustainability. B.B. and J.v.D. are supported by the DFG through the Excellence Cluster "Nanosystems Initiative Munich," Contract No. SFB/TR 12, SFB 631. 


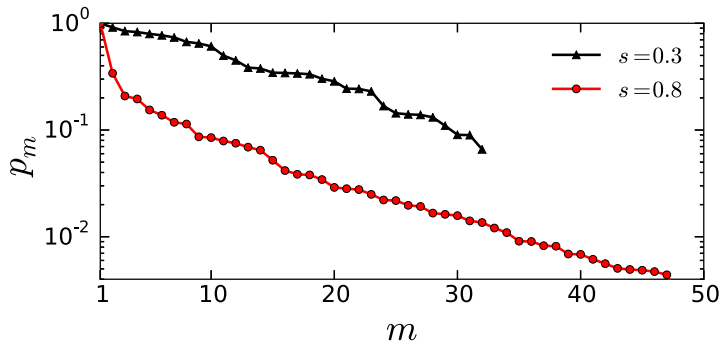

FIG. 8. Weights $p_{m}$ of the coherent state $\left|f^{(m)}\right\rangle$ in the coherentstate expansion (7), as a function of index $m$, for $s=0.3$ (triangles) and $s=0.8$ (circles), with the same parameters as in Fig. 1. The fast exponential decay of the weights illustrates the hierarchical structure of the CSE.

\section{APPENDIX: HIERARCHICAL ALGORITHM FOR THE CSE}

We present here a new algorithm for finding the many-body ground state (7) of the spin-boson model (1), which radically improves the methodology developed previously in Refs. [12] and [13], allowing us to incorporate a large number $M_{\mathrm{cs}}$ of coherent states. This new scheme, devised to optimize efficiently the energy functional, is based only on fast local minimization routines. Indeed, while global minimization routines such as simulated annealing can give the most reliable estimates, they do not scale favorably in the case of a large number of variational parameters. However, blind application of local routines, for instance, L-BFGS or conjugate gradients [38], do not guarantee convergence to the lowest energy minimum. Hence, physical insight must be used as a guide to implement a fast and reliable local optimization method.

Here, we use the fact that the coherent-state decomposition (7) is an expansion that displays a hierarchical structure. Indeed, our simulations demonstrate that the weight $p_{M}$ of a newly added coherent state is typically smaller than the majority of the weights $p_{m}$ of the preceding states. This hierarchical structure is clearly apparent in Fig. 8.

This feature is exploited as follows in our numerical implementation. The algorithm starts with the solution for a single coherent state (the so-called Silbey-Harris ansatz) with $M_{\mathrm{cs}}=1$, which is reliably obtained by a local routine, providing a first estimate of $f_{k}^{(1)}$. Then the energy is minimized for $M_{\mathrm{cs}}=2$ with two coherent states, using the previously determined $f_{k}^{(1)}$ as an initial guess, $f_{k}^{(2)}=0$, and $p_{2}=p_{1} / 2$. Both displacements (and their corresponding weights) are then optimized together. The algorithm continues in the same manner by increasing $M_{\mathrm{cs}}$ by one unit at a time and using the previous displacements and weights as an initial guess for the next minimization stage. For completeness, we give below all the required analytical expressions used in our simulations.

\section{Explicit form of the energy functional}

We focus here on the case of $\mathbb{Z}_{2}$ symmetry, so that the averaged Hamiltonian from the systematic variational state (7) reads

$$
\begin{aligned}
\langle H\rangle= & -\Delta \sum_{n, m=1}^{M_{\mathrm{cs}}} p_{n} p_{m}\left\langle f^{(n)} \mid-f^{(m)}\right\rangle \\
& +\sum_{n, m=1}^{M_{\mathrm{cs}}} p_{n} p_{m}\left\langle f^{(n)} \mid f^{(m)}\right\rangle \sum_{q} 2 \omega_{q} f_{q}^{(n)} f_{q}^{(m)} \\
& -\sum_{n, m=1}^{M_{\mathrm{cs}}} p_{n} p_{m}\left\langle f^{(n)} \mid f^{(m)}\right\rangle \sum_{q} g_{q}\left(f_{q}^{(n)}+f_{q}^{(m)}\right) .
\end{aligned}
$$

The overlaps obey the usual coherent-state algebra (all displacements $f_{q}^{(n)}$ and weights $p_{n}$ are real in the ground state), namely, $\left\langle f^{(n)} \mid f^{(m)}\right\rangle=e^{-(1 / 2) \sum_{q}\left(f_{q}^{(n)}-f_{q}^{(m)}\right)^{2}}$. The minimization is performed on the energy $E=\langle H\rangle / \mathcal{N}$ with the norm $\mathcal{N}=\langle\mathrm{GS} \mid \mathrm{GS}\rangle=2 \sum_{n, m=1}^{M_{\mathrm{cs}}} p_{n} p_{m}\left\langle f^{(n)} \mid f^{(m)}\right\rangle$.

\section{Energy gradients}

Standard optimization routines gain a huge computing advantage by using an explicit expression for the gradient of the function to be minimized. We thus provide here the gradients with respect to the weight $p_{M}$ and displacement $f_{k}^{(M)}$ :

$$
\begin{aligned}
\frac{\partial E}{\partial p_{M}}= & \frac{2}{\mathcal{N}} \sum_{n=1}^{M_{\mathrm{cs}}} p_{n}\left\{-\Delta\left\langle f^{(n)} \mid-f^{(M)}\right\rangle+\left\langle f^{(n)} \mid f^{(M)}\right\rangle\left[\sum_{q}\left[2 \omega_{q} f_{q}^{(n)} f_{q}^{(M)}-g_{q}\left(f_{q}^{(n)}+f_{q}^{(M)}\right)\right]-2 E\right]\right\}, \\
\frac{\partial E}{\partial f_{k}^{(M)}}= & \frac{2 p_{M}}{\mathcal{N}} \sum_{n=1}^{M_{\mathrm{cs}}} p_{n}\left\{\Delta\left\langle f^{(n)} \mid-f^{(M)}\right\rangle\left(f_{k}^{(n)}+f_{k}^{(M)}\right)+\left\langle f^{(n)} \mid f^{(M)}\right\rangle\left(2 \omega_{k} f_{k}^{(n)}-g_{k}\right)\right. \\
& \left.+\left\langle f^{(n)} \mid f^{(M)}\right\rangle\left(f_{k}^{(n)}-f_{k}^{(M)}\right)\left(\sum_{q}\left[2 \omega_{q} f_{q}^{(n)} f_{q}^{(M)}-g_{q}\left(f_{q}^{(n)}+f_{q}^{(M)}\right)\right]-2 E\right)\right\} .
\end{aligned}
$$

[1] S.-K. Ma, Statistical Mechanics (World Scientific, Singapore, 1985).

[2] D. J. Amit, Field Theory, the Renormalization Group, and Critical Phenomena (World Scientific, Singapore, 2005).

[3] K. G. Wilson, Rev. Mod. Phys. 55, 583 (1983).
[4] Understanding Quantum Phase Transitions, edited by Lincoln D. Carr (Taylor and Francis, Boca Raton, FL, 2010).

[5] S. Sachdev, Quantum Phase Transitions (Cambridge University Press, Cambridge, UK, 1999).

[6] R. J. Bartlett and M. Musiał, Rev. Mod. Phys. 79, 291 (2007).

[7] F. Verstraete, J. I. Cirac, and V. Murg, Adv. Phys. 57, 143 (2008). 
[8] U. Schollwoeck, Ann. Phys. (NY) 326, 96 (2011).

[9] S. R. White, Phys. Rev. Lett. 69, 2863 (1992).

[10] U. Schollwöck, Rev. Mod. Phys. 77, 259 (2005).

[11] A. Weichselbaum, F. Verstraete, U. Schollwöck, J. I. Cirac, and J. von Delft, Phys. Rev. B 80, 165117 (2009).

[12] S. Bera, S. Florens, H. U. Baranger, N. Roch, A. Nazir, and A. W. Chin, Phys. Rev. B 89, 121108(R) (2014).

[13] S. Bera, A. Nazir, A. W. Chin, H. U. Baranger, and S. Florens, Phys. Rev. B 90, 075110 (2014).

[14] N. Gheeraert, S. Bera, and S. Florens, arXiv:1601.01545.

[15] S. Florens and I. Snyman, Phys. Rev. B 92, 195106 (2015).

[16] C. Guo, A. Weichselbaum, J. von Delft, and M. Vojta, Phys. Rev. Lett. 108, 160401 (2012).

[17] B. Bruognolo, A. Weichselbaum, C. Guo, J. von Delft, I. Schneider, and M. Vojta, Phys. Rev. B 90, 245130 (2014).

[18] K. E. Cahill, Phys. Rev. 138, B1566 (1965).

[19] N. Zhou, L. Chen, D. Xu, V. Chernyak, and Y. Zhao, Phys. Rev. B 91, 195129 (2015).

[20] L. Duan, S. He, and Q.-H. Chen, arXiv:1412.6343.

[21] A. J. Leggett, S. Chakravarty, A. T. Dorsey, M. P. A. Fisher, A. Garg, and W. Zwerger, Rev. Mod. Phys. 59, 1 (1987).

[22] U. Weiss, Quantum Dissipative Systems (World Scientific, Singapore, 1993).
[23] M. Vojta, Philos. Mag. 86, 1807 (2006).

[24] K. Le Hur, Ann. Phys. (NY) 323, 2208 (2008).

[25] K. Le Hur, Phys. Rev. B 85, 140506 (2012).

[26] M. Goldstein, M. H. Devoret, M. Houzet, and L. I. Glazman, Phys. Rev. Lett. 110, 017002 (2013).

[27] B. Peropadre, D. Zueco, D. Porras, and J. J. García-Ripoll, Phys. Rev. Lett. 111, 243602 (2013).

[28] I. Snyman and S. Florens, Phys. Rev. B 92, 085131 (2015).

[29] R. Bulla, N.-H. Tong, and M. Vojta, Phys. Rev. Lett. 91, 170601 (2003).

[30] R. Bulla, H. J. Lee, N. H. Tong, and M. Vojta, Phys. Rev. B 71, 045122 (2005)

[31] A. Winter, H. Rieger, M. Vojta, and R. Bulla, Phys. Rev. Lett. 102, 030601 (2009).

[32] M. Vojta, R. Bulla, F. Güttge, and F. Anders, Phys. Rev. B 81, 075122 (2010).

[33] A. Freyn and S. Florens, Phys. Rev. B 79, 121102 (2009).

[34] V. J. Emery and A. Luther, Phys. Rev. B 9, 215 (1974).

[35] R. Silbey and R. A. Harris, J. Chem. Phys. 80, 2615 (1984).

[36] A. W. Chin, J. Prior, S. F. Huelga, and M. B. Plenio, Phys. Rev. Lett. 107, 160601 (2011).

[37] I. Snyman and S. Florens (unpublished).

[38] W. W. Hager and H. Zhang, SIAM J. Optimiz. 23, 2150 (2013). 